\title{
Using modern time series analysis techniques to predict ENSO events from the SOI time series
}

\author{
J. I. Salisbury ${ }^{1}$ and M. Wimbush ${ }^{2}$ \\ ${ }^{1}$ Echo Technology, PO. Box 527, Chepachet, RI, USA \\ ${ }^{2}$ Graduate School of Oceanography, University of Rhode Island, Narragansett, RI, USA
}

Received: 16 October 2001 - Revised: 4 December 2001 - Accepted: 8 January 2002

\begin{abstract}
We analyze the monthly 1866-2000 Southern Oscillation Index (SOI) data to determine:

1) whether the SOI data are sufficiently noise-free that useful predictions can be made from them, and

2) in particular, whether future ENSO events can be predicted from the SOI data.
\end{abstract}

The "Hilbert-EMD" technique is used to aid the analysis. This new frequency-time algorithm, based on the Hilbert transform, may be applied to time series for which the conventional assumptions of linearity and stationarity may not apply.

With the aid of the EMD procedure, a cleaner representation of ENSO dynamics is obtained from the SOI data. A polynomial function is then used to predict SOI values. Using only the data from January 1866 through December 1996, this prediction correctly indicated a warm event in 1997-1998 and a cold event in 1999. Using all the data (through December 2000), this prediction shows no strong ENSO events (positive or negative) during the time period January 2001 through December 2004.

\section{Introduction}

El Niño/Southern Oscillation (ENSO) is an interannual climatological disturbance centered on the tropical Pacific; it has global effects and relevance. The term "Southern Oscillation" describes an atmospheric pressure fluctuation centered over the tropical Pacific and Indian Oceans (Philander, 1990). The Southern Oscillation Index (SOI) is sea-level atmosphere pressure (SLP) at Tahiti minus that at Darwin, Australia, normalized as described in the next section. When the SOI is strongly negative, anomalously warm surface waters appear off the coasts of Peru and Ecuador. This is called "El Niño," while the reverse condition with a strong positive SOI and anomalous cold surface waters is called " $\mathrm{La}$ Correspondence to: J. I. Salisbury (echojack@ieee.org)
Niña." Alternatively, these may be called warm and cold ENSO events, respectively. The time interval between successive warm ENSO events ranges from one to eight years and averages 3.6 years.

Poor prediction of ENSO events significantly harms the world economy (Philander, 1990). Conventional models and prediction schemes did not anticipate the large 1986-1987 ENSO event (CPC, 1992). We will show that the dynamics of ENSO are sufficiently represented in the SOI data, so this time series may be usefully employed to predict ENSO events.

In this paper, we use a data analysis method which is not limited to linear, statistically stationary time series. This empirical mode decomposition (EMD) method extracts the energy associated with various intrinsic time scales in generating a collection of intrinsic mode functions (IMF). The IMFs have well-behaved Hilbert transforms, from which instantaneous frequencies can be calculated. Thus, we can localize any event in time as well as frequency. The decomposition can also be viewed as an expansion of the data in terms of the IMFs. Then these IMFs, based on and derived from the data, serve as the basis of that expansion; they can be linear or nonlinear, as dictated by the data. The different IMFs correspond to the different physical time scales which characterize the various dynamical oscillations in the time series.

With our knowledge of ENSO, we can hope to identify an ENSO-related IMF, thus making it possible for prediction techniques to be fruitfully employed to forecast future ENSO events.

\section{Data}

The data set used in this analysis is the monthly- average SOI time series extending from January 1866 through December 2000, with a sampling interval of one month (Mitchell, 1999). This gives a data set of 1620 samples. Figure 1 shows the SOI data from January 1970 to October 2000. If SOI' is normalized sea-level pressure (SLP) at Tahiti minus normal- 


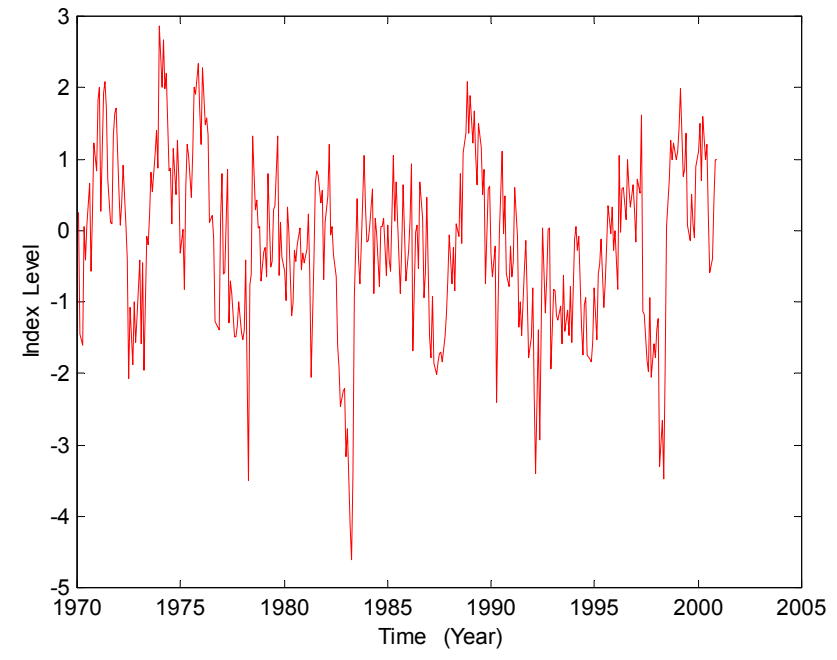

Fig. 1. Monthly SOI data series from January 1970 to October 2000. Tick marks on the time axis indicate the beginning of the designated year.

ized SLP at Darwin, then SOI is SOI' normalized by its standard deviation during 1951-1980. Prior to 1933 there were a few gaps in either the Tahiti or Darwin data; these range in length from 3 to 40 months. Since none of these gaps occur simultaneously at both locations, each gap has been filled by using the normalized pressure at the operating site with the appropriate sign, + for Tahiti, - for Darwin (Ropelewski and Jones, 1987; Allan et al., 1991; Konnen et al., 1998). These filled-in gaps that occurred more than 65 years ago should contribute relatively little to the prediction error.

\section{Hilbert-EMD analysis}

In this paper, we use a new data analysis method known as Empirical Mode Decomposition (EMD), in which a time series is decomposed into a set of intrinsic mode functions (IMF) derived from the data itself (Huang et al., 1998, 1999). The decomposition is based on the direct extraction of energy associated with various intrinsic time scales. The local energy and instantaneous frequency derived from the IMFs through the Hilbert transform can give us a full energyfrequency-time distribution of the data. Such a representation is designated as the Hilbert spectrum; it is well-suited to the analysis of nonlinear and nonstationary data.

With this method, we can localize an event in both time and frequency. The decomposition can be viewed as an expansion of the data in terms of the IMFs. These functions are almost orthogonal, and form a complete basis: the sum of the IMFs equals the original data. The IMFs can be linear or nonlinear, as dictated by the data. Most important of all, the expansion is adaptive. Locality and adaptivity are the necessary features of a basis for expanding nonlinear and nonstationary time series.

To compute an intrinsic mode function from a data set $x(t)$, one first identifies the successive extrema of $x(t)$, then
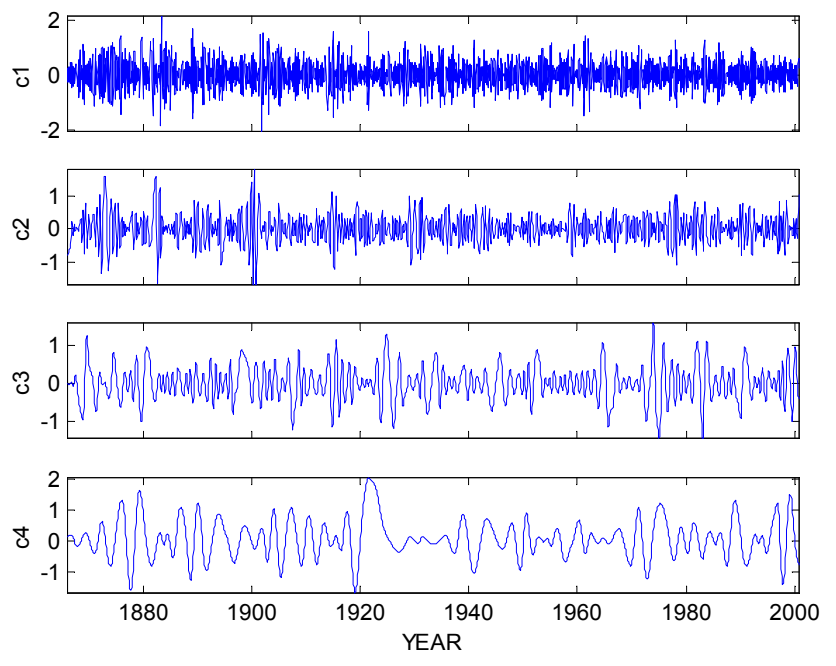

Fig. 2. SOI Intrinsic Mode Functions $C_{1}, C_{2}, C_{3}, C_{4}$ for the SOI data set.
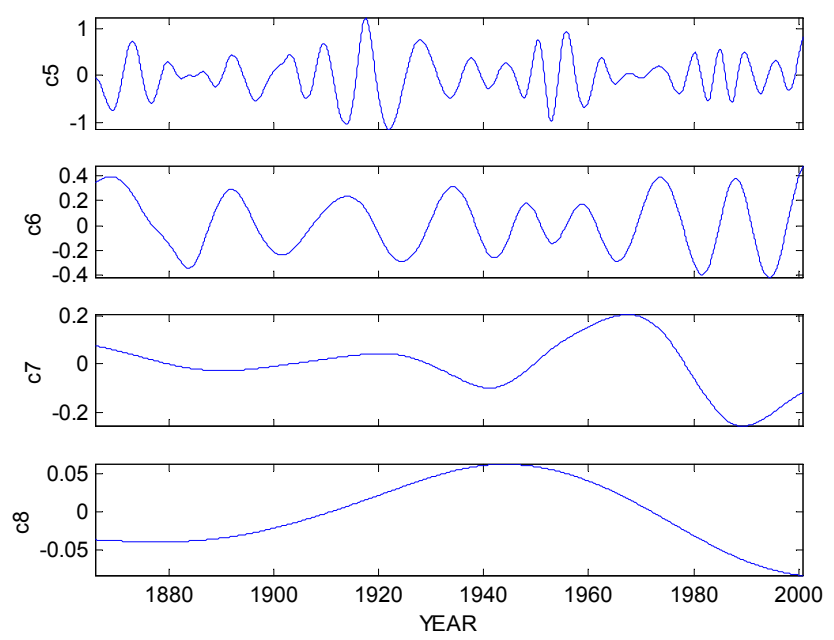

Fig. 3. Same as Fig. 2, but for Intrinsic Mode Functions $C_{5}, C_{6}, C_{7}, C_{8}$.

the local maxima are connected by a cubic spline as the upper envelope, and the local minima are similarly connected as the lower envelope. The mean of these two envelopes is a function of time and designated as $m_{1}(t)$. The difference between the data $x(t)$ and the mean $m_{1}(t)$ is computed and designated $h_{1}(t)=x(t)-m_{1}(t)$. This $h(t)$ is approximately the first IMF. To determine it more accurately, we treat $h_{1}(t)$ as a new set of data, determine its upper and lower envelopes and compute their new mean, $m_{11}(t)$, as well as the difference $h_{11}(t)=h_{1}(t)-m_{11}(t)$. This $h_{11}(t)$ is again treated as a new data set, and the process, referred to as "sifting," is repeated a number of times. The sifting process is stopped when the number of zero-crossings of $h_{1 k}$ equals the number of extrema. The convergent result is designated by $C_{1}(t)$, which is the first IMF of the data set $x(t)$; it has a zero local mean.

Now subtract $C_{1}(t)$ from the data set $x(t)$, and call the 
difference, "the first residue":

$R_{1}(t)=x(t)-C_{1}(t)$

The residue is analyzed by the same method as if it were new data. A new mean is found and the difference, $R_{1}(t)$ minus its mean, converges to a function of time, $C_{2}(t)$, which is the second intrinsic mode function of $x(t)$. It also has a zero local mean. The second residue,

$R_{2}(t)=R_{1}(t)-C_{2}(t)$

is formed, and the process is continued until either $C_{n}$ or $R_{n}$ becomes so small that it is less than a predetermined value of substantial consequence, or the residue $R_{n}$ becomes a monotonic function from which no more IMFs can be extracted.

In the real world, the data set to be analyzed is generally noisy, nonstationary and of limited duration. These three factors greatly limit our ability to distinguish whether, for example, the dynamical system is a random process, a low-order noisy chaotic system or a high-order chaotic system. If the signal of interest is captured in a single IMF, we can examine the predictability of this IMF.

The IMFs for the SOI data, computed using the sifting process described above, are presented in Figs. 2 and 3. $C_{9}$ is the final residue; it shows a monotone trend. As can be seen, $C_{1}$ is composed of the smallest time scales, or highest frequencies, and the time scale increases as the index $i$ of $C_{i}$ increases. IMFs 1 through 5 contain the major portion of the energy in the SOI signal.

A few cycles of the Hilbert transforms of IMFs 3 and 4 are presented in Figs. 4 and 5. Intrawave modulation can be observed in both these figures. This behaviour is characteristic of nonlinear dynamics. Also, note that IMF 4 has a characteristic frequency of $0.03 \mathrm{cpm}$, which roughly corresponds to the 3.6-year mean interval between ENSO warm events. Figure 6 presents a comparison of the SOI and IMF 4 data for the years January 1970 to October 2000. IMF 4 resembles a smoothed version of the SOI data. We will attempt to use both the SOI time series and the IMF 4 series to predict ENSO events.

\section{Prediction}

We must now select an effective dynamical model, which will allow us to predict the evolution of any new point in the phase space within the limits of the intrinsic instabilities embodied in the data. The basic idea is that since we know approximately how points in a neighborhood evolve into points in the next neighborhood, we can make a predictive estimate using an appropriate function, $G$, such that

$\hat{\zeta}(n+1)=G(\zeta(n))$.

Using the techniques described by Kantz and Schreiber (1997), various functions for predicting future events of nonlinear dynamical systems were employed on the SOI and IMF 4 data. Various options, input parameter values and prediction models were tried. Evaluation was performed by
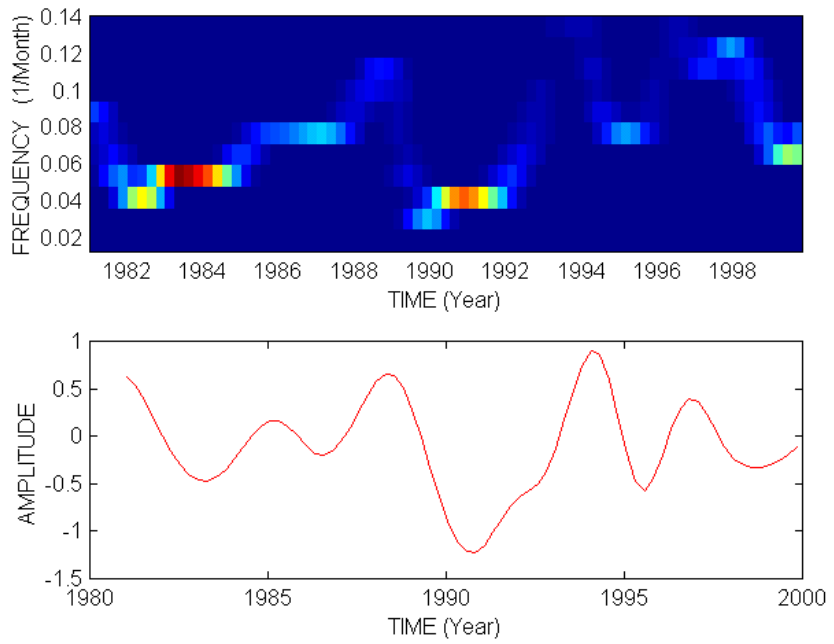

Fig. 4. The Hilbert spectrum (upper panel) from IMF 3 (lower panel) of the SOI for the period 1982-2000. Note the intrawave modulation in which the local frequency varies within the dominant cycle of the oscillation.
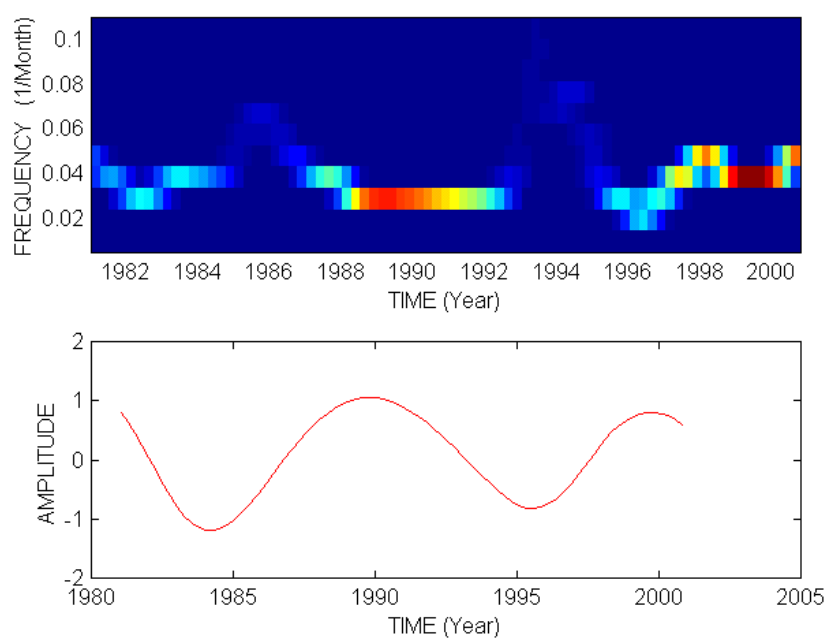

Fig. 5. Same as Fig. 4, but for IMF 4.

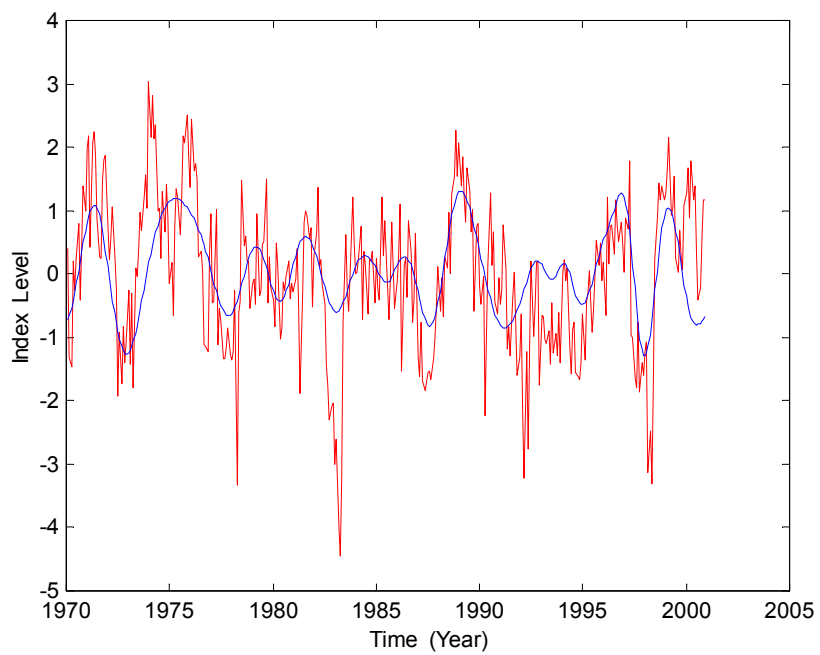

Fig. 6. Monthly SOI data series (red) from January 1970 to October 2000. Also, the IMF 4 data series for the same time period. Tick marks on the time axis indicate the beginning of the designated year. 


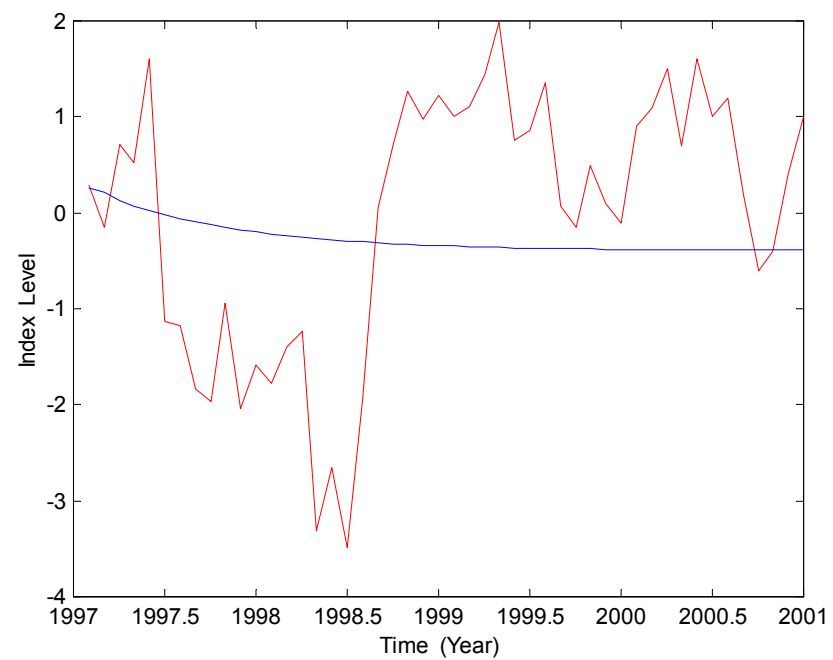

Fig. 7. 48-month prediction (blue) based on the measured SOI data prior to 1997. Actual SOI time series for the 1997-2000 period of the prediction is also shown (red).

looking at both the in-sample and out-of-sample errors. We selected the prediction algorithm and the combination of embedding dimension and delay resulting in the smallest outof-sample error. The functional form selected, which is flexible enough to model the true function on the whole attractor, is a multivariate polynomial (Giona et al., 1991), which for degree $h$ and dimension $d$ has $k=(d+h) ! /(d ! h !)$ independent coefficients. The problem to be solved, given the $N d$-dimensional vectors

$(x(1), x(2), \ldots, x(N))$,

is to reconstruct the mapping,

$\boldsymbol{x}(n+1)=\boldsymbol{F}(\boldsymbol{x}(n))$,

where $\boldsymbol{F}$ is a $d$-dimensional vector of polynomial functions of the $d$ components of $\boldsymbol{x}$.

The combination of an embedding dimension of 4, a delay of 1 month, and a third-degree polynomial, gave the smallest out-of-sample error. All techniques and parameters resulted in predicted variations occurring at a slightly lower frequency than those observed.

We first used the SOI data without the last 48 data points, January 1997 to December 2000, to make a prediction for these four years. The period from January 1866 to December 1996 was used as a training set to predict 48 months into the future. The results are depicted in Fig. 7, which shows a nearly useless prediction (blue), giving a proper value only for the mean, as expected for a random process.

Next, the Hilbert-EMD procedure was carried out on the SOI data to obtain IMF 4 for the period January 1866 - December 1994, and again for the period January 1866 - December 1995. The same scheme was used to predict the succeeding 48 month period in each case, January 1995 - Decemeber 1998 (Fig. 8) and January 1996 - December 1999 (Fig. 9). Both of these predictions show a strong "cold event"

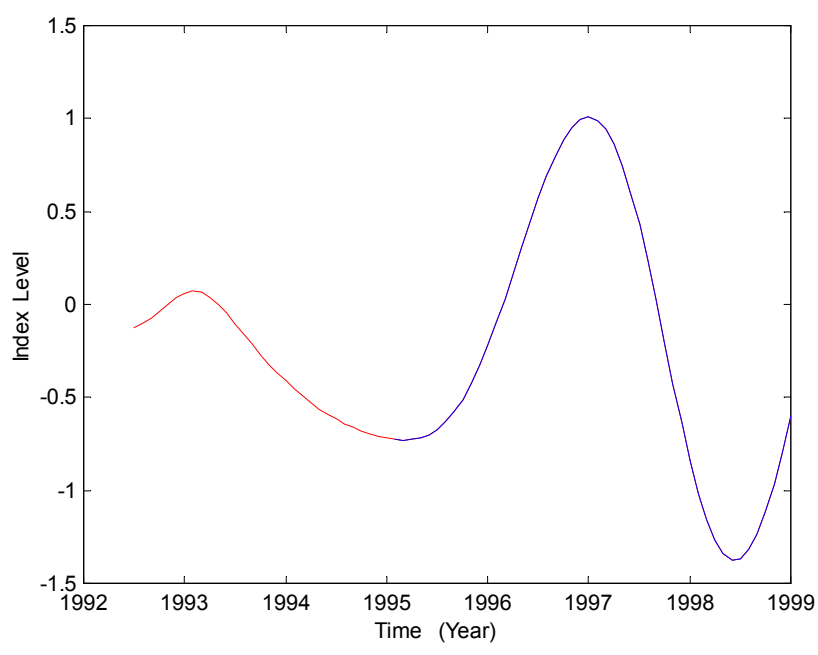

Fig. 8. 48-month prediction (blue) of IMF 4 computed from SOI data prior to 1995 (red).

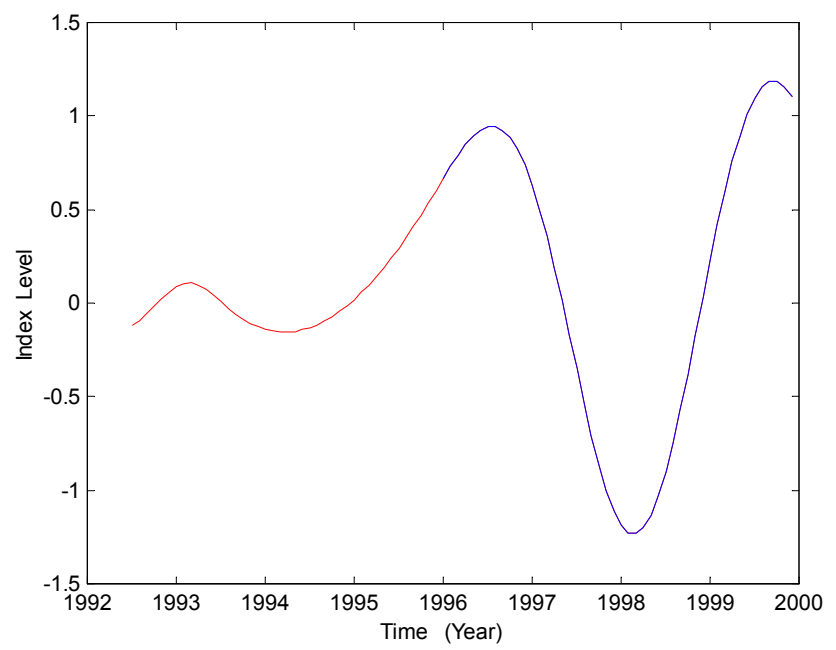

Fig. 9. 48-month prediction (blue) of IMF 4 computed from SOI data prior to 1996 (red).

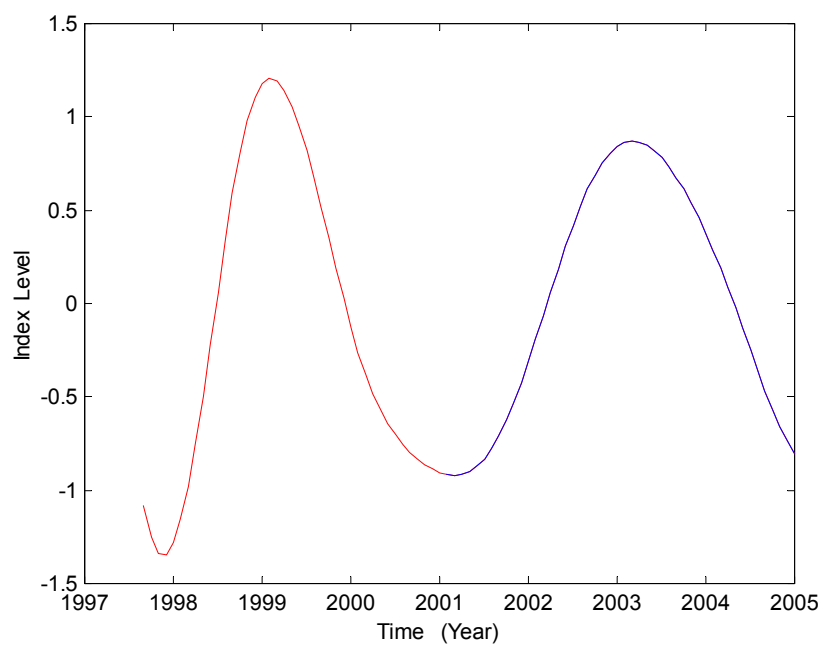

Fig. 10. 48-month (2001-2004) prediction (blue) of IMF 4 computed from SOI data prior to 2001 (red). 
(SOI $>1$ ) followed by a strong "warm event" $(\mathrm{SOI}<-1)$, and for the second case, which extends through the year 2000, another strong "cold event" (SOI $>1)$. This agrees with the observed SOI index (Fig. 7, red curve), except that the predicted times for these events are somewhat later than the observed times, as a result of the frequency shift mentioned above. Now using the IMF 4 data derived from all available SOI data up to December 2000, a 48-month, January 2001 - December 2004 prediction was made; it is presented in Fig. 10. The actual measured data, including the past warm and cold events, are plotted (red) along with the predicted (blue) results, which begin after the last known data point (December 2000). The predicted SOI magnitudes from January 2001 to December 2004 are all less than one, indicating that no strong warm or cold events are predicted by this procedure before December 2004.

\section{Conclusion}

It has been shown that the Empirical Mode Decomposition separates the total dynamics in the SOI into a finite number of simpler components. Attempting to predict future events using the undecomposed data proved fruitless. By analyzing just one of the EMD components, IMF 4, which has the same mean frequency as ENSO, we have been able to study a much simpler dynamical system and predict future events.

Out-of-sample predictions based on the 4th Intrinsic Mode Function of the SOI data show a strong warm event in 19971998 and a strong cold event in 1999, as observed. They also forecast no strong ENSO events during the period 20012004.

\section{References}

Allan, R. J., Nicholls, N., Jones, P. D., and Butterworth, I. J.: A further extension of the Tahiti-Darwin SOI, early ENSO events and Darwin pressure, J. Climate, 4, 743-749, 1991.

CPC (Climate Prediction Center, NOAA): Experimental Long-Lead Forecast Bulletin, 6, No. 1, March, 1992.

Giona, M., Lentini, F., and Cimagalli, V.: Functional reconstruction and local prediction of chaotic time series, Phys. Rev. A, 44, 3496-3502, 1991.

Huang, N. E., Shen, Z., Long, S. R., Wu, M. C., Shih, H.-H., Zheng, Q., Yen, N.-C., Tung, C.-C., and Liu, H.-H.: The empirical mode decomposition and the Hilbert spectrum for nonlinear and nonstationary time series ananlysis, Proc. Roy. Soc. London Ser. A, 454, 903-995, 1998.

Huang, N. E., Shen, Z., and Long, S. R.: A new view of nonlinear water waves: the Hilbert spectrum, Annual Review of Fluid Mechanics, 31, 417-457, 1999.

Kantz, H. and Schreiber, T.: Nonlinear Time Series Analysis, Cambridge University Press, 1997.

Konnen, G. P., Jones, P. D., Kaltofen, M. H., and Allan, R. J.: Pre1866 extensions of the Southern Oscillation Index using early Indonesian and Tahitian meteorological readings, Journal of Climate, 11, 2325-2339, 1998.

Mitchell, T.: Southern Oscillation Index, http://tao.atmos . washington.edu/pacs/additional_analyses/ soi.html, 1999.

Philander, S. G.: El Niño, La Niña, and the Southern Oscillation, Academic Press, San Diego, 1990.

Ropelewski, C. F. and Jones, P. D.: An extension of the TahitiDarwin Southern Oscillation Index, Monthly Weather Review, 115, 2161-2165, 1997. 